\title{
Fruit drop in two kiwifruit varieties and the use of two Bacillus-based biofungicides
}

\author{
Seona Casonato \\ Lincoln University, Pest Management and Conservation Department, PO Box 5084, Lincoln \\ 7647, New Zealand \\ seona.casonato@lincoln.ac.nz
}

\begin{abstract}
Recently, fruit drop in two green varieties of kiwifruit (Actinidia deliciosa; VarA and $\operatorname{VarB}$ ) has increased towards the harvest date. The efficacy of two biofungicides, applied post-flowering, to ameliorate the effects of early fruit drop in VarA and VarB during the 2017-18 growing season was tested. Treatments were applied to a single bay, with buffer bays and rows adjacent. Treatments were two different Bacillus-based biofungicide products; Serenade $\operatorname{Max}$ (a.i. B. subtilis QST713 strain) and Triple-X ${ }^{\oplus}$ (a.i. B. amyloliquefaciens BS 1b). There was an untreated control. All fruits in the canopy, within the treated bay, were counted and recorded at 4 -weekly intervals, over 5 months until harvest. At the VarA site, there was no statistical difference $(\mathrm{P}>0.1)$ in the percentage of fruit drop between the control $(7 \%)$, Triple- $\mathrm{X}^{\varpi}(5 \%)$ and Serenade ${ }^{\varpi}$ Max (10\%) treated vines. At the VarB site, fruit drop differed statistically $(\mathrm{P}<0.1)$ between Triple- $\mathrm{X}^{\oplus}$ treated vines $(5 \%)$ and the control $(10.5 \%)$, with Serenade ${ }^{\oplus}$ Max treated vines (6.5\%) having intermediate fruit drop. The use of Triple- $\mathrm{X}^{\circledast}$ may be an option to assist with reducing fruit drop in kiwifruit.
\end{abstract}

Keywords: kiwifruit, fruit drop, biofungicides, Bacillus

\section{INTRODUCTION}

In recent times, fruit drop in some varieties of kiwifruit (Actinidia deliciosa), in particular two green varieties (due to commercial confidentiality called VarA and VarB from this point forward) has increased towards the harvest date. Dispensation has been given by Zespri International to growers affected by fruit drop to allow an earlier harvest by requiring reduced Brix and number of black seeds. The severity of fruit drop can fluctuate from season to season, and the precise cause behind the phenomenon is unknown. In previous work, several species of fungi have been found in vines exhibiting fruit drop. The presence of these fungi has not been identified as the causal agent of the fruit drop, but rather it is suggested that various factors predispose the vines to fungal infection, which could potentially contribute to the early fruit drop. Various studies have investigated the use of products containing Bacillus spp. for the control of fruit drop (e.g. Li et al. 2013 for apples) and previous research on kiwifruit has indicated that fruit drop was lessened in orchards with the use of Bacillus-based biological products (Casonato unpublished data). Bacillus-based products have also been linked to not only plant protection, but also growth stimulation (Perez-Garcia et al. 2011). These bacteria can promote plant health by stimulating nutrition and growth and, as such, can improve the overall health of A. deliciosa vines.

Thus, the premise of the current trials was to ascertain whether two biofungicides, Serenade ${ }^{\star}$ Max (a.i. Bacillus subtilis; Bayer Crop Science New Zealand) and Triple- $\mathrm{X}^{\oplus}$ (a.i. Bacillus amyloliquefaciens; Biostart, Auckland), could reduce the amount of fruit drop in the current growing season on two different green varieties of kiwifruit in orchards that had previously experienced fruit drop. This would be achieved by potentially reducing fungal infections that 
may contribute to fruit drop and improving overall plant health. These trials were not to ascertain the causal agent for fruit drop, but to provide a treatment that could ameliorate fruit drop close to harvest.

\section{MATERIALS AND METHODS}

\section{VarA and VarB orchards}

Both kiwifruit orchards used for the trials were located near Pongakawa in the Bay of Plenty, New Zealand and received the same treatments post-flowering, with normal orchard management being undertaken by the orchardist. The treatments were a control (no biofungicide application; TRT1) and two different biofungicide products with differing Bacillus spp. as the active ingredient: Triple- $\mathrm{X}^{\oplus}$ (a.i. B. amyloliquefaciens BS 1b; TRT2) Serenade ${ }^{\varpi}$ Max (a.i. B. subtilis QST 713 strain; TRT3). Each trial was set up with a row of treated vines and a row of non-treated vines in the adjacent row that acted as a buffer when spraying was undertaken. Treatments were applied to a single bay, with bays and rows adjacent to the treatment bay acting as a buffer zones (Figs. 1 \& 2).
All spraying was done by a commercial spraying company at approximately four-weekly intervals, post-flower emergence, dependent on the weather conditions. Spraying was undertaken with a commercial sprayer, Fantini ECO 2000 (Andreoli Engineering, Moderna, Italy) with a twin-ring spray set up. The sprayer was calibrated for $1000 \mathrm{~L} / \mathrm{ha}$ on $3.5 \mathrm{~m}$ rows at the VarA orchard and $3.2 \mathrm{~m}$ rows at the VarB orchard. Each of the biofungicides and control was applied with the surfactant and ultra-violet protectant $\mathrm{Nu}$ Film (Miller Corp., USA) added at $500 \mathrm{~mL} / \mathrm{ha}$. Biofungicide, Serenade Max was applied a 3000 g/ha whilst Triple-X was applied at $3000 \mathrm{~mL} / \mathrm{ha}$. The spray tank, pump and nozzles were triple rinsed with fresh water before use and between each spray application. Each product was precisely measured using calibrated scales and measuring jugs. There was a $200-\mathrm{L}$ buffer in the spray tank for each application to eliminate air being sucked into the tank.

\section{VarA orchard}

The VarA orchard was on a relatively flat site. The orchard was well maintained and vines have

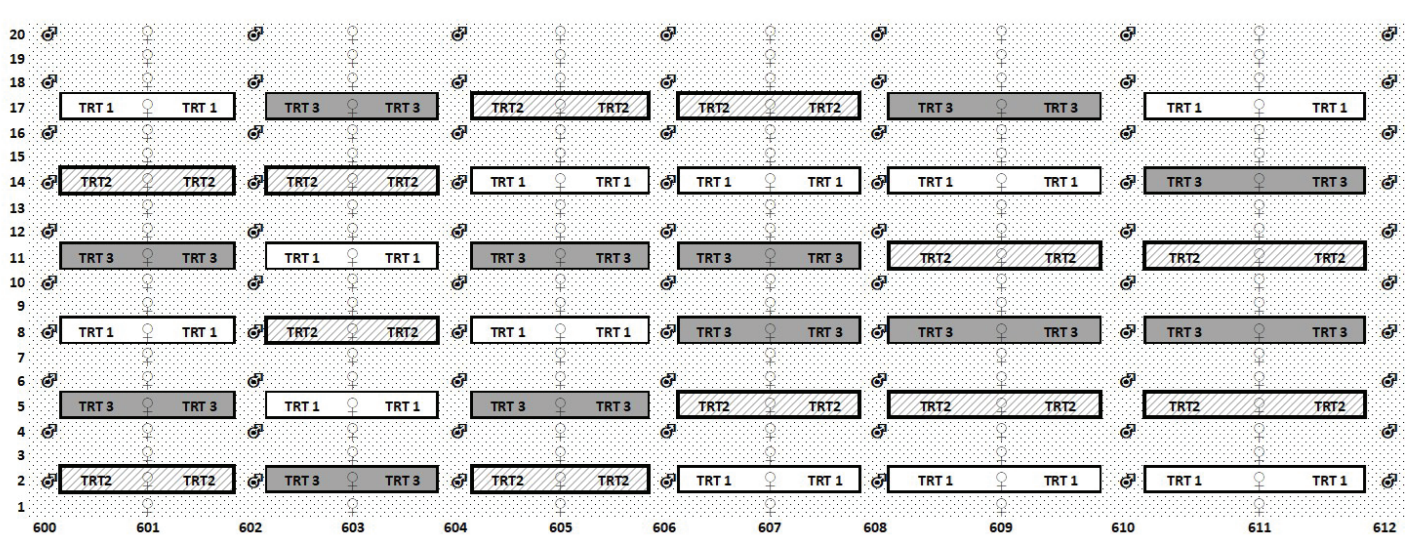

Figure 1 Layout of the VarA orchard trial to investigate the efficacy of two biofungicides. Treatments: Control (no spray, TRT1), Triple-X ${ }^{\circledast}$ (a.i. Bacillus amyloliquefaciens, TRT2) and Serenade ${ }^{\circledR}$ Max (a.i. Bacillus subtilis, TRT3). Male vines - $\sigma^{\top}$ Female vine - ㅇ․ Numbers 600 to 612 on the bottom of the figure represent the rows within the orchard. Number 1 to 20 on the far left of the figure indicate the bays. Each treatment bay (represented by TRT1, TRT2 or TRT3 and surrounded by a thick black border) was the entire bay (left and right) of the female row vine where fruit counts were taken. All other bays within the trial were buffers (indicated by dots). 


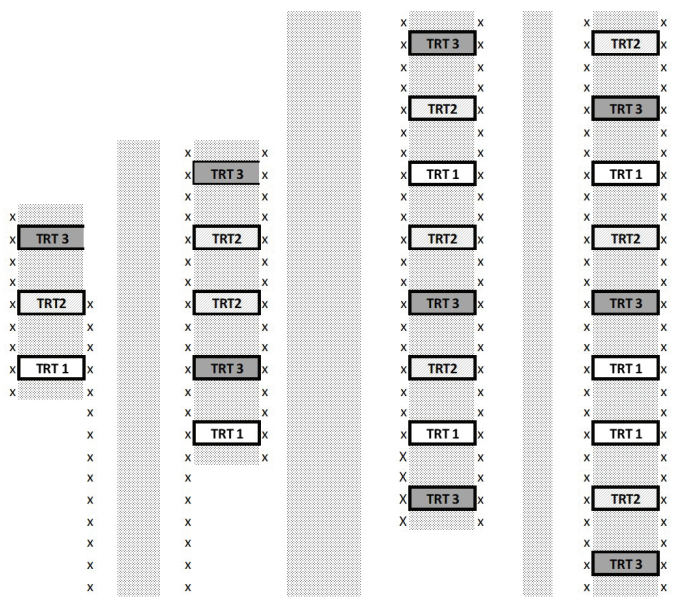

Figure 2 Layout of the VarB orchard trial to investigate the efficacy of two biofungicides. Treatments: Control (no spray, TRT1 $\mathrm{n}=7$ ), Triple- $\mathrm{X}^{\oplus}$ (a.i. Bacillus amyloliquefaciens, TRT2 $\mathrm{n}=9$ ) and Serenade ${ }^{\varpi}$ Max (a.i. Bacillus subtilis, TRT3 $n=9$ ). All other bays within the trial were buffers (indicated by dots). Vines indicated by X.

established well, providing good cane coverage within a bay. The orchard block was set up with strip males between the female rows (Fig. 1). For the trial, 20 bays were used per row, with two complete replicates per row. The trial layout was a fully replicated block design with a total of twelve replicate bays (Fig. 1). In each treatment bay, both to the left and the right of the treated vine(s) (Fig. 1) had all fruitlets/fruit within the canopy counted and recorded at 4-weekly intervals until harvest.

The trial area (treatment and control vines) was also under normal management provided by the owners. The following products were applied: Kasumin, a bactericide for the management of Pseudomonas syringae pv. actinidiae (Psa) (4 October 2017), Defence 500 - Rovral (a.i. iprodione) (18 November 2017), copper (21 November 2017) and Tech Urea (12 December 2017). Trial spraying with the Bacillus spp. products was undertaken on the following dates: 29 November 2017, 21 December 2017, 17 January 2018, 13 February 2018 and 6 March 2018.
All fruit in the canopy within the treated bay were counted and recorded, thus enabling the determination of fruit loss between counts. Fruit counts were undertaken on three occasions: 19 January 2018, 20 February 2018 and 17 March 2018. A visual assessment was undertaken at the time of counting and the following characteristics recorded: callusing of canes and girdling $(0=$ no callusing; $1=$ partial callusing; $2=$ complete callusing, for the entire vine): the health of the rootstock (given an arbitrary "good" or "bad" criteria, where "good" was given when there was a good scion/rootstock union, no major wounds on the rootstock and the leader was healthy or "bad", whereby the scion/rootstock union was poor, there was rotting material in the rootstock and the scion leader had no major cracking or wounding); and any other unusual visible symptoms, that appeared to veer from normal growth. At each assessment date, samples of fruit that fell off during the process of counting and fruit that remained intact on the cane, were returned to the laboratory for culturing on potato dextrose agar (PDA) plates. The pedicel and the entire fruit were surface sterilised in $5 \%$ bleach solution prior to plating. Samples of fruit flesh, fruit skin and the entire pedicel were plated. Samples of crumbling or abnormal rootstocks were also taken to the laboratory for culturing and identification via morphological characteristics and molecular amplification of the internal transcribed spacer region.

\section{VarB orchard}

The VarB orchard was located on an undulating site. The site had the VarB scions grafted to rootstocks that exhibited signs of wood decay. The male vines were intra-row with the females (Fig. 2). Due to the limited number of VarB vines available, the trial was set-up as a block design with unequal replication. No chemical sprays had been applied to this orchard prior to the treatment schedule. Trial spraying with the Bacillus spp. products was undertaken on the following dates: 29 November 2017, 21 December 2017, 17 January 2018, 13 February 2018, 6 March 2018, 4 April 2018 and 3 May 2018. 
Fruit counts were undertaken on: 26 January 2018, 27 February 2018, 26 March 2018, 9 April 2018, 29 April 2018 and 10 May 2018. All assessments were undertaken as per VarA orchard.

\section{Statistical analysis}

All data were checked for normality and transformed when necessary for normal distribution to be achieved. Residuals were also checked for normality. Analysis of variance (ANOVA) or restricted maximum likelihood (REML) models were undertaken on the data in Genstat version 18 (VSI International). When a significant F-test was obtained for treatments, data were subjected to means separation by a Bonferroni test. Significance was accepted at $\mathrm{P}<0.1$ as this was a preliminary trial with variability, such as different rootstocks, that was unable to be controlled.

\section{RESULTS}

\section{VarA orchard}

Fruit were harvested by the orchard on 26 March 2018 for packhouse delivery. Prior to harvest, the final fruit count on 17 March 2018, indicated there was a substantial amount of variability in the total number of kiwifruit that had dropped. The degree of fruit drop varied greatly within a row, appearing to be linked to the contour of the row with higher areas in the row having more visible fruit drop compared with areas lower in the row. Fruit drop appeared to be affected by the quality of the rootstock, particularly whether any visible wood-rotting fungus was present and whether any wounding sites, being girdling or cane cutting, had callused or not. These variables were factored into the analysis.

During the 2017-18 growing season, there was minimal fruit drop during January and February although the number of dropped fruit varied greatly within each treatment. By midMarch the fruit began to fall and the numbers of fallen fruit increased.

At the VarA trial site, there was no statistical difference $(\mathrm{P}>0.1)$ in the number of fruit drop between the control and either of the treatments

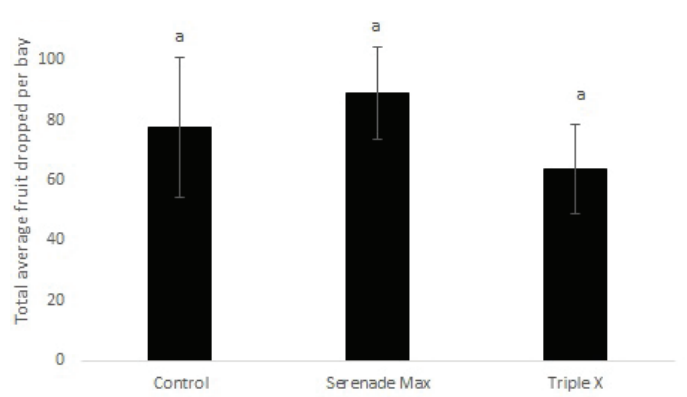

Figure 3 The mean number of fruit per bay dropped in the two treatments, Serenade $\operatorname{Max}^{\infty}$ and Triple- $\mathrm{X}^{\circledast}$, compared to the control that received no biofungicide spray at the VarA kiwifruit orchard. Bars represent standard error of the mean. Letters above the columns indicate significance at $10 \%$ (mean separation by a Bonferroni test).

(Fig. 3). The average total fruit dropped was greater in the Serenade Max treated bays in comparison to the Triple-X and control vines. This equated to approximately $7 \%, 5 \%$ and $10 \%$ of the average total fruit drop per bay for the control, Triple-X and Serenade Max treated vines, respectively.

Visual assessment of all the treatment vines indicated there was a difference in the amount of healing between vines after girdling or cane cutting. Vines showed wounds that were either completely or partially healed or not healed at all. There was a significant $(\mathrm{P}=0.014)$ interaction between the amount of callus exhibited by a vine and the treatment that was applied to the vine. When the vine did not produce callus, the Serenade Max treatment had significantly $(\mathrm{P}=0.014)$ more fruit drop compared to the Triple-X and control treated vines. When the vine formed calluses that had completely or partially developed, the Serenade Max treated vines had fruit drop that was not different from either the Triple-X treatment or the control.

The rootstock where fruit drop occurred, often exhibited signs of wood decay. Hypochnicium lyndoniae was found in association with 
deteriorating rootstocks and a Phytophthora sp. was also isolated from the root/soil zone of the trunk. Unfortunately, the culture became contaminated with Pythium species prior to molecular confirmation.

Cultures from fruit and their associated stalks that dropped at the time of assessment and those intact on the vine, indicated that the fungus Botryosphaeria dothidea was more commonly associated with those that had dropped. Botryosphaeria spp. were also isolated from some cane wounds. Kiwifruit intact on the cane had very little fungi present and any recovery was of either Phomopsis spp. or Fusarium spp. The sample number taken was small and, as such, no statistical indication or correlation can be made.

\section{VarB orchard}

Fruit were harvested from VarB orchard on 10 May 2018 and there was substantial variability in the total number of kiwifruit that dropped. This variation appeared, superficially, to be dependent on the position within the row and also the health of the rootstock (data not shown). Commonly isolated from unhealthy rootstocks at this orchard were Hypochnicium lyndoniae and Phellinus species. Botryosphaeria spp. were consistently isolated from fruit that had dropped.

There was a significant difference at $(\mathrm{P}=0.088)$ between the total number of fruit that dropped per bay in the control (no product) and the biological product Triple-X (Fig. 4). Use of the B. subtilis product, Serenade Max, did not differ significantly from either the control or Triple-X. Vines treated with B. amyloliquefaciens (Triple-X) had nearly $60 \%$ less fruit drop than untreated control vines. The proportion of fruit lost at the time of harvest, compared to the initial number of fruit on the cane within the bay, indicates a significant difference $(\mathrm{P}=0.063)$ between the control and both of the treatments. The control vines lost over $10 \%(\bar{x}=97)$ of the fruit within a bay due to fruit drop compared to $6.5 \% \quad(\bar{x}=57)$ and $5 \%(\bar{x}=39)$ for Serenade Max and Triple-X treated vines respectively.

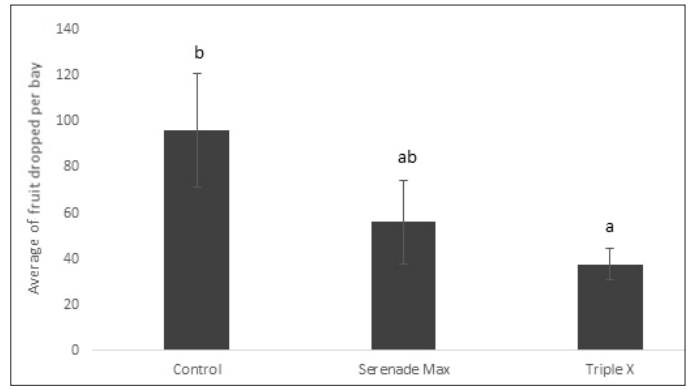

Figure 4 The mean number of fruit per bay dropped in the two treatments, Serenade $\operatorname{Max}^{\circ}$ and Triple- $\mathrm{X}^{\oplus}$, compared to the control that received no biofungicide spray at the $\operatorname{VarB}$ kiwifruit orchard. Bars represent standard error of the mean. Letters above the columns indicate significance at $10 \%$ (mean separation by a Bonferroni test).

\section{DISCUSSION}

Kiwifruit vines treated with Triple-X had the least fruit drop with $5 \%$ for the season, at both VarA and VarB orchards. There is potential for Triple-X to be used to reduce fruit drop in the two green kiwifruit varieties trialled. However, this research has indicated there is variability between vines and in the efficacy of the biofungicides in regard to fruit drop. Possible reasons for these results include kiwifruit variety, orchard management practices and the health of rootstocks. Overall, there was no significant difference in the number of fruit dropping between both Bacillus-based biofungicides and the control (non-treated vines) in the VarA orchard.

The lack of clearly significant results among treatments at the VarA orchard could be due to the pre-flowering chemical treatments affecting the efficacy of the biofungicides. The application of copper can have a deleterious effect on $B$. subtilis and could affect the colonisation ability of the biofungicide on the kiwifruit vines surface (van Eeden \& Korsten 2004). No pre-flowering sprays were used at the VarB orchard. In avocado (Persea americana) production, it has been found that the copper product, Coprox Super (Arysta LifeScience, SA), had a negative effect on the 
growth of B. subtilis and, as such, the effect of different copper compounds and the timing intervals between applications in an integrated programme have been investigated (van Eeden \& Korsten 2004). At the VarA orchard, the copper application was made 8 days prior to the first application of the biofungicides and it is very likely this had a negative impact in the Bacillus spp. colonisation ability. Future work should be undertaken to determine appropriate time intervals between biofungicides and other chemical sprays when used as part of an integrated management programme for kiwifruit.

At the VarA orchard, both scion girdling and cane cutting had occurred prior to the application of the trial treatments and as such it appears that the Serenade Max treated vines may have been in poorer health due to the open wounds, potentially exposing them to fungal infection, and consequently this may be reflected in greater fruit drop. For callus formation in plants, there is a close association with auxin and cytokinin production (Ikeuchi et al. 2013). Bacillus spp. have been identified to be directly involved in the modulation of phytohormones and can produce auxin that can stimulate nutrient uptake (PérezGarcía et al. 2011). Bacillus amyloliquefaciens has been shown to biosynthesise indole-3-acetic acid (IAA), which is responsible for plant growth promotion. Additionally, B. subtilis produces cytokinins that have a beneficial effect on plant growth (Idris et al. 2007; Pérez-García et al. 2011) and indirectly the formation of callus. Thus, it would be useful to undertake a trial to determine if an application of Bacillus-based biofungicides at the time of wounding (i.e. girdling and cane cutting) had an effect on callus production. That is, the use of Bacillus spp. products could not only induce callus formation but could potentially protect the vine from fungal infection through the wounds.

The rootstock where fruit drop occurred often showed symptoms of wood decay and had a scion/rootstock union that had not developed well. Commonly isolated from these vines were Hypochnicium lyndoniae and Phellinus spp. These are both genera associated with wood rot. These fungi are distributed worldwide and have been found previously in New Zealand. Research in Italy has indicated that Fomitiporia mediterraneae (now known as Phellinus mediterraneae) was isolated from white rot in kiwifruit rootstock, which have light-coloured and crumbling wood (Osti \& Di Marco 2014). The presence of these decay fungi may affect the overall health of the vine. Bacillus amyloliquefaciens (a.i. of Triple X) contributes to soil biofertilisation by improving bioavailability of essential compounds and increasing the supply of mineral nutrients, such as phosphorus, to the vine (Perez-Garcia et al. 2011).

This trial was not designed to ascertain the causal agent for fruit drop that is experienced at orchards growing VarA and VarB, but to provide a treatment that could ameliorate the loss of kiwifruit within orchards close to harvest. These trials have indicated the application of Triple- X, a biofungicide containing B. amyloliquefaciens, does reduce the incidence of fruit drop in treated vines in green kiwifruit varieties. This is a valuable tool as part of an integrated management strategy within the orchard. This trial has also indicated that the health of the rootstock may play a role in the ability of biofungicides to be efficacious. The potentially complex interactions among several factors influencing vine health and the influence of these factors on the vine's response to spray treatments suggested by this research (including fruit drop and other factors), highlights the need for careful vine selection in future trials if these effects are to be teased out and their interactions correctly identified. In future, these trials should be taken over a larger scale and at various stages of fruit development. Sprays of the biofungicides at flowering are worth investigating and further work must be undertaken to move away from calendar spraying.

\section{ACKNOWLEDGEMENTS}

This project was funded by Zespri International. The author would like to thank Shvonn Cunningham and Garry Hill for technical assistance. Many thanks to the anonymous reviewer of the paper for useful comments. 


\section{REFERENCES}

Idris EE, Inglesia DJ, Talon $\mathrm{M}$, Borriss $\mathrm{R}$ 2007. Tryptophan-dependent production of indole-3-actic acid (IAA) affects level of plant growth promotion by Bacillus amyloliquefaciens FZB42. Molecular PlantMicrobe Interactions 20: 619-629.

Ikeuchi M, Sugimoto K, Iwase A 2013. Plant callus: mechanisms of induction and repression. The Plant Cell 25: 3159-3173.

Li Y, Han LR, Zhang Y, Fu X, Chen X, Zhang L, Mei R, Wang Q 2013. Biological control of apple ring rot on fruit by Bacillus amyloliquefaciens 9001. Plant Pathology Journal 29:168-173.

Osti F, Di Marco S 2014. Correlations between soil sodicity and foliar symptoms of wood decay of kiwifruit. Journal of Plant Pathology 96: 121-131.

Pérez-García A, Romero D, de Vicente A 2011. Plant protection and growth stimulation by microorganisms: biotechnological applications of Bacilli in agriculture. Current Opinion in Biotechnology 22: 187-193.

Tugizimana F, Mhlongo MI, Piater LA, Dubery IA. 2018. Metabolomics in plant priming research: the way forward? International Journal of Molecular Sciences 19, e1759.

Van Eeden M, Korsten L 2004. Effect of additives and copper fungicide on Bacillus subtilis to control avocado (Persea americana Mill.) fruit disease. South African Avocado Growers' Association Yearbook 27: 11-16. 